\title{
Glucagon-like peptide 1-related peptides increase nitric oxide effects to reduce platelet activation
}

\author{
Cristina Barale ${ }^{1,2}$; Simona Buracco2; Franco Cavalot ${ }^{1,2}$; Chiara Frascaroli, ${ }^{1,2}$; Angelo Guerrasio ${ }^{1,2}$; Isabella Russo ${ }^{1,2}$ \\ ${ }^{1}$ Internal Medicine and Metabolic Disease Unit, San Luigi Gonzaga Hospital, Turin, Italy; ${ }^{2}$ Department of Clinical and Biological Sciences of the Turin University, Turin, Italy
}

\begin{abstract}
Summary
Glucagon-like peptide 1 (GLP-1) is object of intensive investigation for not only its metabolic effects but also the protective vascular actions. Since platelets exert a primary role in the pathogenesis of atherosclerosis, inflammation and vascular complications, we investigated whether GLP-1 directly influences platelet reactivity. For this purpose, in platelets from 72 healthy volunteers we evaluated GLP-1 receptor (GLP-1R) expression and the effects of a 15-minute incubation with the native form GLP-1(7-36), the N-terminally truncated form GLP-1(9-36) and the GLP-1 analogue Liraglutide (100 nmol/l) on: i) aggregation induced by collagen or arachidonic acid (AA); ii) platelet function under shear stress; iii) CGMP and CAMP synthesis and CGMPdependent protein kinase (PKG)-induced Vasodilator-StimulatedPhosphoprotein (VASP) phosphorylation; iv) activation of the signalling molecules Phosphatidylinositol 3-Kinase (PI3-K)/Akt and Mitogen Activated Protein Kinase (MAPK)/ERK-1/2; and v) oxidative stress. Ex-
\end{abstract}

Correspondence to:

Dr. Isabella Russo, PhD

Internal Medicine and Metabolic Disease Unit

Department of Clinical and Biological Sciences of the Turin University

San Luigi Gonzaga Hospital

10043 Orbassano (Turin), Italy

Tel.: + 39011 9026622, Fax: + 390119038639

E-mail: isabella.russo@unito.it periments were repeated in the presence of the nitric oxide donor $\mathrm{Na}$-nitroprusside. We found that platelets constitutively express GLP-1R and that, independently of GLP-1R, GLP-1 (7-36), GLP-1(9-36) and Liraglutide exert platelet inhibitory effects as shown by: a) increased NO-antiaggregating effects, b) increased the activation of the cGMP/PKG/VASP pathway, c) reduced the activation of PI3-K/Akt and MAPK/ERK-2 pathways, d) reduced the AA-induced oxidative stress. When the experiments were repeated in the presence of the antagonist of GLP-1R Exendin(9-39), the platelet inhibitory effects were maintained, thus indicating a mechanism independent of GLP-1R. In conclusion, GLP-1(7-36), its degradation product GLP-1(9-36) and Liraglutide exert similar inhibitory effects on platelet activation, suggesting a potential protective effect on the cardiovascular system.

\section{Keywords}

Platelets, glucagone-like peptide 1, Liraglutide, nitric oxide, diabetes

Financial support:

This study was supported by a grant from the Italian Ministry of Education, University and Research (MIUR), Projects of National Interest (PRIN 2012).

Received: July 29, 2016

Accepted after major revision: March 19, 2017

Epub ahead of print: April 13, 2017

https://doi.org/10.1160/TH16-07-0586

Thromb Haemost 2017; 117: 115-1128

\section{Introduction}

The incretin hormone Glucagon-like peptide 1 (GLP-1), exerts a pivotal role in the maintenance of glucose homeostasis (1). It is principally secreted as GLP-1(7-36) amide, the biologically active circulating GLP-1 metabolite which, after binding to a specific GLP-1 receptor (GLP-1R), induces the increase of glucose-stimulated insulin secretion. The biologic half-life of GLP-1(7-36) is very short since after secretion it is rapidly degraded by the enzyme dipeptidyl peptidase (DPP)-4 to GLP-1(9-36), the main GLP-1 metabolite in vivo which does not interact with GLP-1R (1).

In type 2 diabetes the incretin effect is deeply reduced (2) and the GLP-1-based therapies are now established as treatment options for the disease. Since type 2 diabetes therapy aims not only to improve the glycaemic homeostasis but also to prevent vascular complications, it is of the utmost interest to investigate if the "new drugs" introduced in clinical practice, like incretin mimetics, have a valuable impact on cardiovascular disease.

Interestingly, multiple actions of GLP-1 and the structurally related GLP-1R agonists are exerted on cells and tissues that do not express the classical GLP-1R (3). The evidence that the GLP-1(9-36) metabolite is also able to exert beneficial effects on the cardiovascular system suggests that some actions of the native GLP-1 could be exerted through a mechanism independent of GLP-1R (4). Actually, GLP-1(9-36) exerts cardiovascular actions like cardioprotection against the ischaemia-reperfusion injury, vasodilation (5-7), and activation of the nitric oxide (NO)/guanosine-3',5'-cyclic monophosphate (cGMP) pathway in vascular endothelium (8). Thus, GLP-1(9-36) cannot be considered an inactivated' form of GLP-1 as previously thought.

Furthermore, previous studies suggest that GLP-1 metabolites and its long-acting analogues are able to decrease oxidative stress by increasing intracellular antioxidant defences and decreasing the reactive oxygen species (ROS) production through GLP-1R-dependent and -independent pathways (9). Therefore, there is a strong rationale to verify whether GLP-1 influences platelets, cells deeply involved in the pathogenesis of atherosclerosis (10), par- 
ticularly of diabetic vascular complications, thus justifying a role for antiplatelet therapy both in secondary and in primary prevention in diabetes mellitus (11).

The adhesion/aggregation process is regulated by the balance between pro- and anti-aggregating circulating agents such as NO. As well known, NO is involved in platelet anti-aggregation through the synthesis of cGMP and the consequent activation of the cGMP-dependent protein kinase (PKG): the cGMP/PKG system modulates basic mechanisms of platelet activation (12). Inhibitory effects exerted by exenatide alone - but not by either the native GLP-1(7-36) or the truncated GLP-1(9-36) - have been recently shown in megakaryocyte cell line and platelets suggesting the ability of this GLP-1R agonist to directly influence platelet function (13).

However, the inability of GLP-1(7-36) and GLP-1(9-36) to influence per se platelet aggregation cannot exclude the possibility that GLP-1 peptides interfere with physiological antiaggregating agents such as NO and intracellular pathways that potentially could influence the cardiovascular system also by means of the platelet response.

Furthermore, the aim to specifically investigate the role of metabolically-inactive GLP-1(9-36) on cardiovascular function may contribute to explain the apparently disappointing results from some large-scale DPP-4 inhibitor trials that failed to demonstrate any benefit on cardiovascular outcomes in patients affected by diabetes $(14,15)$, considering a possible reduced action of GLP-1(7-36) in addition to the inhibited production of GLP-1(9-36).

The present study was designed to examine the ability of GLP-1 to influence in vitro platelet responses, their reactivity to $\mathrm{NO}$ and signalling molecules stimulated by pro-aggregating agents. We tested the effects of the native GLP-1(7-36), of the N-terminally truncated GLP-1(9-36) form and of the GLP-1 analogue Liraglutide.

\section{Materials and methods}

\section{Chemicals}

Collagen and arachidonic acid (AA) were purchased from Mascia Brunelli Spa (Monza, Milan, Italy). GLP-1(7-36), GLP-1(9-36) were obtained from DBA Italia Srl (Segrate, Milan, Italy). Liraglutide (VICTOZA ${ }^{\circ}$ ) was obtained from Novo Nordisk (Glostrup, Denmark). The sources of the specific antibodies are shown in the different sections. The other reagents were obtained from Sigma (St. Louis, MO, USA). In the experiments, unless specified, we used $100 \mathrm{nmol} / \mathrm{l}$ for GLP-1(7-36), GLP-1(9-36) and Liraglutide. This concentration is about to 5-10 times the concentrations detectable in incretin-based therapy (16) and, as known, higher drug concentrations are needed to obtain effects in vitro similar to those in vivo.

\section{Subjects and platelet preparation}

Seventy-two healthy volunteers ( 40 men/32 women; age: $24.6 \pm 0.4$ years; body mass index [BMI]: $22.7 \pm 0.7 \mathrm{~kg} / \mathrm{m}^{2}$ ) were studied. The subjects were non smokers, normotensive, did not take any drug in the previous two weeks and showed normal range values also after oral glucose tolerance test. All exhibited normal insulin sensitivity (HOMA-IR: $1.7 \pm 0.05)$. They gave informed consent before investigation and the Ethics Committee of our Hospital approved the study.

Fasting venous blood samples were anticoagulated with $3.8 \%$ trisodium citrate (v/v: 1/9) or citrate-dextrose solution (ACD; v/v: 1/6). Platelet-rich plasma (PRP) was obtained by using Platelet Function Centrifuge (BioData Corporation, Horsham, PA, USA). To prepare washed platelets (WP), ACD-anticoagulated PRP was processed as previously described (17).

\section{Evaluation of GLP-1R}

To evaluate the presence of GLP-1R on platelet surface flow cytometry assays and western blot analyses were performed. WP were fixed, permeabilised and labelled with phycoerythrin (PE)-conjugated mouse monoclonal anti-Human GLP-1R (R\&D Systems, Minneapolis, MN, USA). As a negative control, a PE Mouse IgG2b isotype control antibody (BioLegend, San Diego, CA, USA) was used at the same concentration as the (PE)-conjugated mouse monoclonal anti-Human GLP-1R. In addition, WP were labelled with allophycocyanin (APC)-conjugated mouse monoclonal antiHuman Integrin a2b/CD41 (R\&D Systems) as positive control for platelet identification. Analysis was carried out by using CyAn ADP Analyzer (Beckman Coulter, Brea, CA, USA).

For western blot analysis, WP samples were centrifuged and pellets dissolved in Laemmly buffer and processed as previously described (17). Membranes were incubated with mouse antibody anti-human GLP-1R and goat anti-Vinculin (Santa Cruz Biotechnology, Santa Cruz, CA, USA). As secondary antibodies, we used, respectively, goat anti-mouse (Jackson ImmunoResearch, West Grove, PA, USA) and donkey anti-goat (SantaCruz Biotechnology) conjugated to horseradish peroxidase. Blots were scanned and analysed densitometrically by the image analyzer 1D Image Analysis software (Kodak, Rochester, NY, USA). Pancreatic tissue (Santa Cruz Biotechnology) was used as positive control.

\section{Platelet aggregation studies}

Platelet aggregation studies were carried out in PRP by following light-scattering changes as described by Born (18) using a eightchannel aggregation system (Platelet Aggregation Profiler, Model PAP-8, BioData Corporation). Platelet aggregation tests were carried out after a 15-minute ( $\mathrm{min}$ ) preincubation with GLP-1(7-36), GLP-1(9-36) and Liraglutide with or without SNP (5 $\mu \mathrm{mol} / 1,5$ $\mathrm{min})$ and induced by AA $(0.5 \mathrm{mmol} / \mathrm{l})$ or collagen $(4 \mathrm{mg} / \mathrm{l})$. Each aggregation test was recorded for $5 \mathrm{~min}$ and reported as maximal aggregation (MA).

\section{Platelet reactivity in high shear-stress conditions}

Whole blood (WB) samples were exposed to GLP-1(7-36), GLP-1(9-36), Liraglutide and platelet reactivity was evaluated by Platelet Function Analyzer (PFA-100, Siemens Healthcare Diag- 
nostic Products GmbH, Marburg, Germany) (19), measuring the time needed to form a platelet plug named "closure time" (seconds), within an aperture cut into a platelet-reactive membrane coated with collagen plus epinephrine (CEPI). Tests were carried out also in the presence of SNP $(20 \mu \mathrm{mol} / \mathrm{l}, 5 \mathrm{~min})$.

\section{CGMP and CAMP production}

cGMP and cAMP were measured in PRP samples $(500 \mu \mathrm{l})$ both in the absence and in the presence of a 20 -min preincubation with the phosphodiesterase inhibitor 3-isobutyl-1-methylxanthine (IBMX) (100 $\mu \mathrm{mol} / \mathrm{l})$, then incubated with GLP-1(7-36), GLP-1(9-36), or Liraglutide for $15 \mathrm{~min}$ with or without SNP (5 $\mu \mathrm{mol} / \mathrm{l}, 5 \mathrm{~min})$. Platelet samples were processed as previously described (20). Some experiments were repeated after a preincubation with the $\mathrm{NO}$ synthase (NOS) inhibitor $\mathrm{N}^{\mathrm{G}}$-monomethylL-arginine (L-NMMA) (1 mmol/l, $20 \mathrm{~min})$, the soluble Guanylate Cyclase (sGC) inhibitor methylene blue (MB) $(50 \mu \mathrm{mol} / \mathrm{l}, 20 \mathrm{~min})$, or after exposure to AA $(100 \mu \mathrm{mol} / 1,8 \mathrm{~min})$ with or without preincubation with NADPH-oxidase inhibitor apocynin $(10 \mu \mathrm{mol} / \mathrm{l}, 20$ $\min )$.

cGMP and cAMP were determined using cGMP and cAMP EIA kits (Abnova, Taipei City, Taiwan) according to the manufacturer's instructions.

\section{Signalling transduction molecule detection}

To evaluate the influence of GLP-1 on the PKG/VASP pathway activated by NO, WP were exposed to GLP-1(7-36), GLP-1(9-36) or Liraglutide, then stimulated by SNP ( $5 \mu \mathrm{mol} / \mathrm{l}, 5 \mathrm{~min})$. To evaluate GLP-1 influence on PI3-K and MAPK pathways activation, WP were incubated with GLP-1(7-36), GLP-1(9-36) or Liraglutide, then activated by collagen $(4 \mathrm{mg} / \mathrm{l})$ or AA $(100 \mu \mathrm{mol} / \mathrm{l})$. Some experiments were repeated after a 20-min preincubation with the GLP-1R antagonist Exendin(9-39) (100 nmol/l), the PKG inhibitor KT5823 (1 $\mu \mathrm{mol} / \mathrm{l})$ and the protein kinase A (PKA) inhibitor PKI $(10 \mu \mathrm{mol} / \mathrm{l})$. Then, samples were processed as described above and membranes incubated with the following antibodies: mouse anti-Akt, rabbit anti-phosphoAkt ${ }^{\mathrm{Ser}-473}$ and mouse anti-phosphoERK-1/2 ${ }^{\text {Tyr-204 }}$,(Santa Cruz Biotechnology), rabbit anti-ERK1/2 (R\&D Systems) and mouse anti-phosphoVASPser-239 (Calbiochem-Merck Millipore, Darmstadt, Germany). As secondary antibodies we used goat anti-mouse and goat anti-rabbit (Santa Cruz Biotechnology).

\section{ROS production}

Intracellular ROS were evaluated in WP by using the sensitive fluorescent indicator 2',7'-dihydrodichlorofluoresceine diacetate (DCF-DA), oxidised by $\mathrm{H}_{2} \mathrm{O}_{2}$ to the highly fluorescent DCF (21). WP $\left(6 \times 10^{7} \mathrm{ml}^{-1}\right)$ were exposed to $10 \mu \mathrm{mol} / \mathrm{l} \mathrm{DCF}-\mathrm{DA}$ and incubated for 15 min with GLP-1(7-36), GLP-1(9-36) or Liraglutide. $\mathrm{AA}$ was added just before measuring fluorescence. To examine the requirement for AA signalling events in ROS generation, platelets were preincubated for 20 min with the PI3-K inhibitors wortman- $\operatorname{nin}(100 \mathrm{nmol} / \mathrm{l})$ and LY294002 $(100 \mu \mathrm{mol} / \mathrm{l})$, the selective MAPK/ ERK-1/2 inhibitor U0126 (40 $\mu \mathrm{mol} / \mathrm{l})$, and the NADPH-oxidase inhibitors apocynin $(10 \mu \mathrm{mol} / \mathrm{l})$ and diphenyleniodonium (DPI) $(10 \mu \mathrm{mol} / \mathrm{l})$ and then exposed to GLP-1-related peptides. In addition, platelets were incubated with dihydroethidium (DHE) (20 $\mu \mathrm{mol} / \mathrm{l})$, pelleted, rinsed, dissolved in phosphate-buffered saline, supplemented with calcium and fluorescence was measured to evaluate superoxide production.

Fluorescence was measured over a 60 -min period at 1 -min intervals using a plate fluorometer (GloMax-Multi Detection System, Promega Corporation, Madison, WI, USA) fitted with $490 \mathrm{~nm}$ excitation and $520 \mathrm{~nm}$ emission filters. Fluorescence per minute was first calculated for each sample, then ROS production was expressed as fold increase over basal values.

\section{Statistical analysis}

All values in the text and figures are expressed as mean \pm SEM. Data were analysed using Student's t-test, or one-way ANOVA followed by post-hoc Bonferroni's test. Results were significant when $\mathrm{p}<0.05$.

\section{Results \\ Expression of GLP-1R}

As shown in Figure 1, analyses revealed appreciable levels of GLP-1R expression on platelet surface: actually, GLP-1R was detected in 60 to $78 \%$ (mean: $71.5 \pm 1.8 \%$ ) of platelets by flow cytometry assay $(n=15)(A)$. Isotype control for the GLP1R antibody did not stain the sample.

Western blot $(n=10)(B)$ showed that GLP-1R, expressed in pancreatic tissue as positive control, was consistently expressed also in platelets.

\section{GLP-1 influence on platelet aggregation in PRP}

In response to collagen ( $>$ Figure $2 \mathrm{~A}$ ) or AA ( $>$ Figure $2 \mathrm{~B}$ ) the antiaggregating effect of SNP was increased by GLP-1(7-36) $(\mathrm{n}=24)$, GLP-1 $(9-36)(\mathrm{n}=24)$, and Liraglutide $(\mathrm{n}=24)$.

Notably, the GLP-1(7-36), GLP-1(9-36) and Liraglutide actions on the antiaggregating effects of SNP did not differ when platelets were preincubated with $\operatorname{Exendin}(9-39)(n=24, p=N S$ for each), thus indicating that the ability of GLP-1(7-36), GLP-1(9-36) and Liraglutide to increase the antiaggregating effects of SNP occurred independently of GLP-1R. Quantitatively, after exposure to SNP: i) the aggregation to collagen decreased by $34 \pm 5 \%$ and the addition of GLP-1(7-36), GLP-1(9-36) and Liraglutide effectively decreased platelet aggregation by $59 \pm 3 \%, 57 \pm$ $3 \%$ and $56 \pm 3 \%$, respectively; ii) the aggregation to AA decreased by $36 \pm 5 \%$ with SNP, and the addition of GLP-1(7-36), GLP-1(9-36) and Liraglutide decreased platelet aggregation by 48 $\pm 3 \%, 49 \pm 5 \%$ and $48 \pm 4 \%$, respectively. Notably, the GLP-1(7-36), GLP-1(9-36) and Liraglutide actions on the antiaggregating effects of SNP did not differ when platelets were prein- 


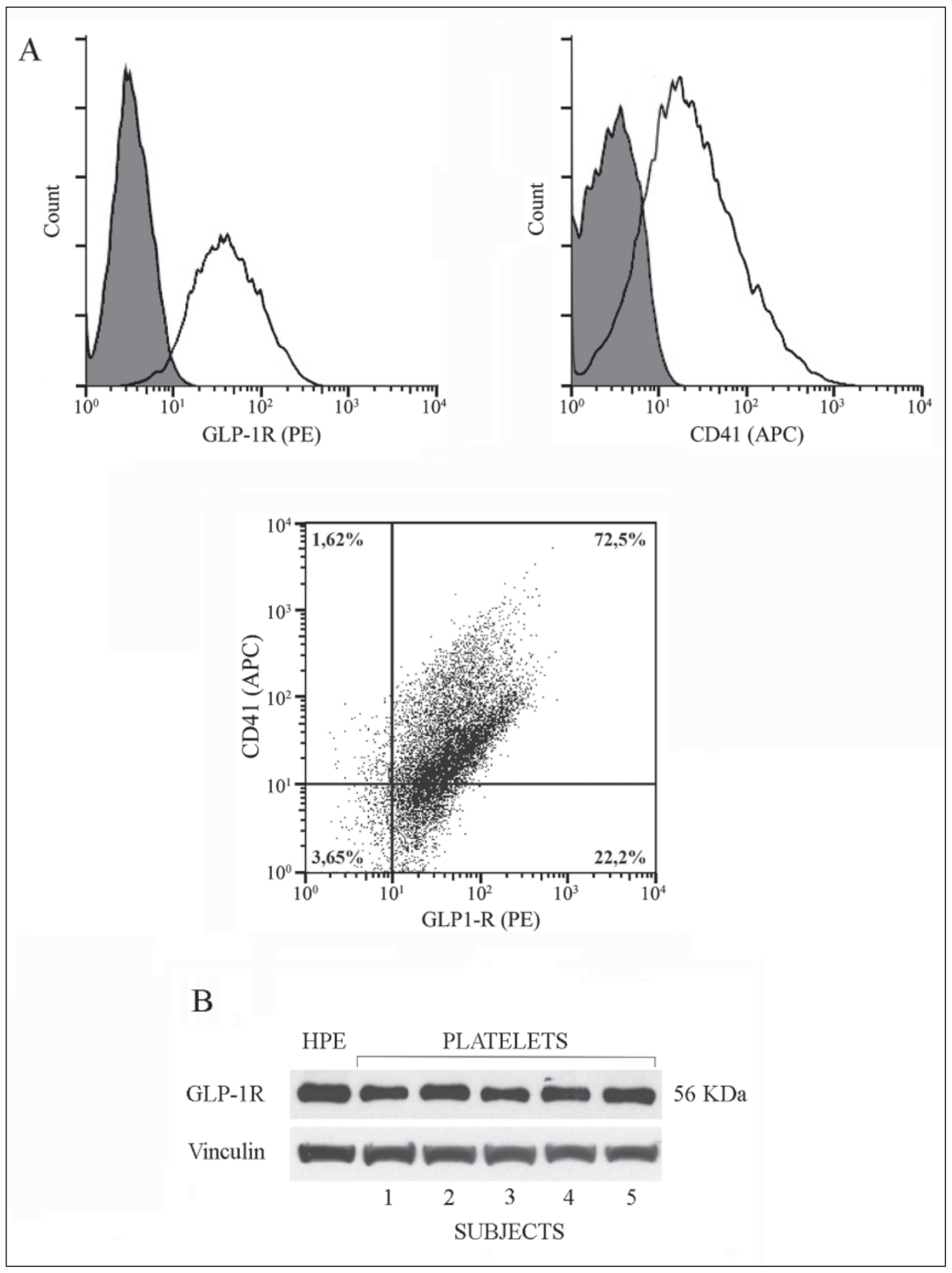

Figure 1: Expression of GLP-1R on platelet surface. Platelets were stained by PE-conjugated mouse anti-human GLP-1R specific monoclonal antibody and APCconjugated mouse monoclonal anti human integrin $a 2 b / C D 41$, and FACS assay analysis was performed. The figure shows the single labelling for GLP-1R (PE) or CD41 (APC) and the double labelling dot plot GLP1-R(PE) + CD41(APC)) $(n=15)$ (A). Western blot analysis of platelet samples from five different subjects and of human pancreatic exstract (HPE) sample as positive control. Blot is representative of platelet samples obtained from 10 subjects (B).

cubated with the GLP-1R antagonist Exendin(9-39) ( $\mathrm{n}=24, \mathrm{p}=\mathrm{NS}$ for each), thus indicating that the ability of GLP-1(7-36), GLP-1(9-36) and Liraglutide to increase the antiaggregating effects of SNP occurred independently of GLP-1R.

GLP-1 peptides alone did not modify platelet aggregation triggered by collagen ( $84 \pm 5$ vs $91 \pm 3$ with GLP-1(7-36), vs $80 \pm 4$ with GLP-1(9-36), vs $91 \pm 4$ with Liraglutide, $n=12$, $p=N S$ for all) or by AA ( $86 \pm 6$ vs $82 \pm 6$ with GLP-1(7-36), vs $81 \pm 5$ with GLP-1(9-36), vs $92 \pm 4$ with Liraglutide, $n=12$, $p=N S$ for all). Furthermore, we also examined GLP-1 alone effects on platelet aggregation at shorter time points (3-7 $\mathrm{min}$ ) and lower concentrations $(1-10 \mathrm{nmol} / \mathrm{l})$ and no inhibitory effects were found (data not shown). 
Figure 2: GLP-1 effects on platelet aggregation and PFA-100.

Platelets were exposed for $15 \mathrm{~min}$ to GLP-1(7-36),

GLP-1(9-36) and Liraglutide $(100 \mathrm{nmol} / \mathrm{l})$ and their influence was evaluated on the antiaggregating effects of the NO donor SNP $(5 \mu \mathrm{mol} / \mathrm{l})$ in response to collagen (4 $\mathrm{mg} / \mathrm{l})(\mathrm{A})$ and $\mathrm{AA}(0.5$ $\mathrm{mmol} / \mathrm{l})(B)(\mathrm{n}=24$ per group) or PFA closure time with CEPI (collagenepinephrine cartridges) $(n=6)(C)$, both in the absence and presence of the GLP-1 receptor antagonist Exendin(9-39) (Exe) (100 nmol/l). ANOVA: $\mathrm{p}<0.0001$, Bonferroni's post-test: ${ }^{*} p<0.05$ vs SNP alone, \# $p<0.05$ vs basal.

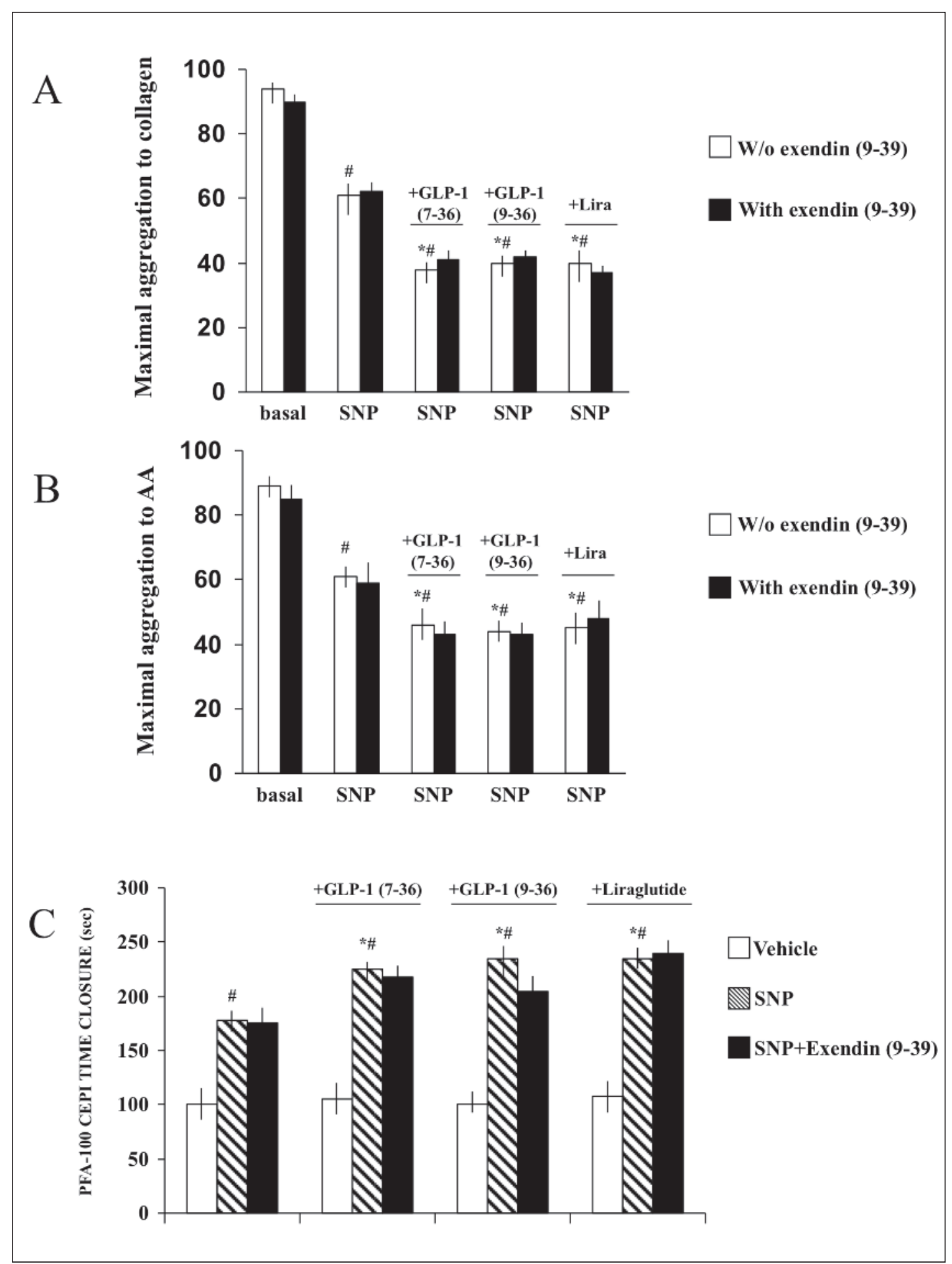

\section{GLP-1 influence on platelet function evaluated by PFA-100 in WB}

Figure 2C shows the GLP-1(7-36), GLP-1(9-36) and Liraglutide effects on platelet adhesion and aggregation in WB measured by CEPI PFA-100. GLP-1-related peptides alone did not modify closure time. However, closure time was prolonged by SNP and it was further significantly increased when WB samples were preincubated with GLP-1(7-36), GLP-1(9-36), and Liraglutide $(n=6)$. The pretreatment with Exendin(9-39) did not alter the effects of GLP-1 peptides on closure time prolongation induced by SNP. 


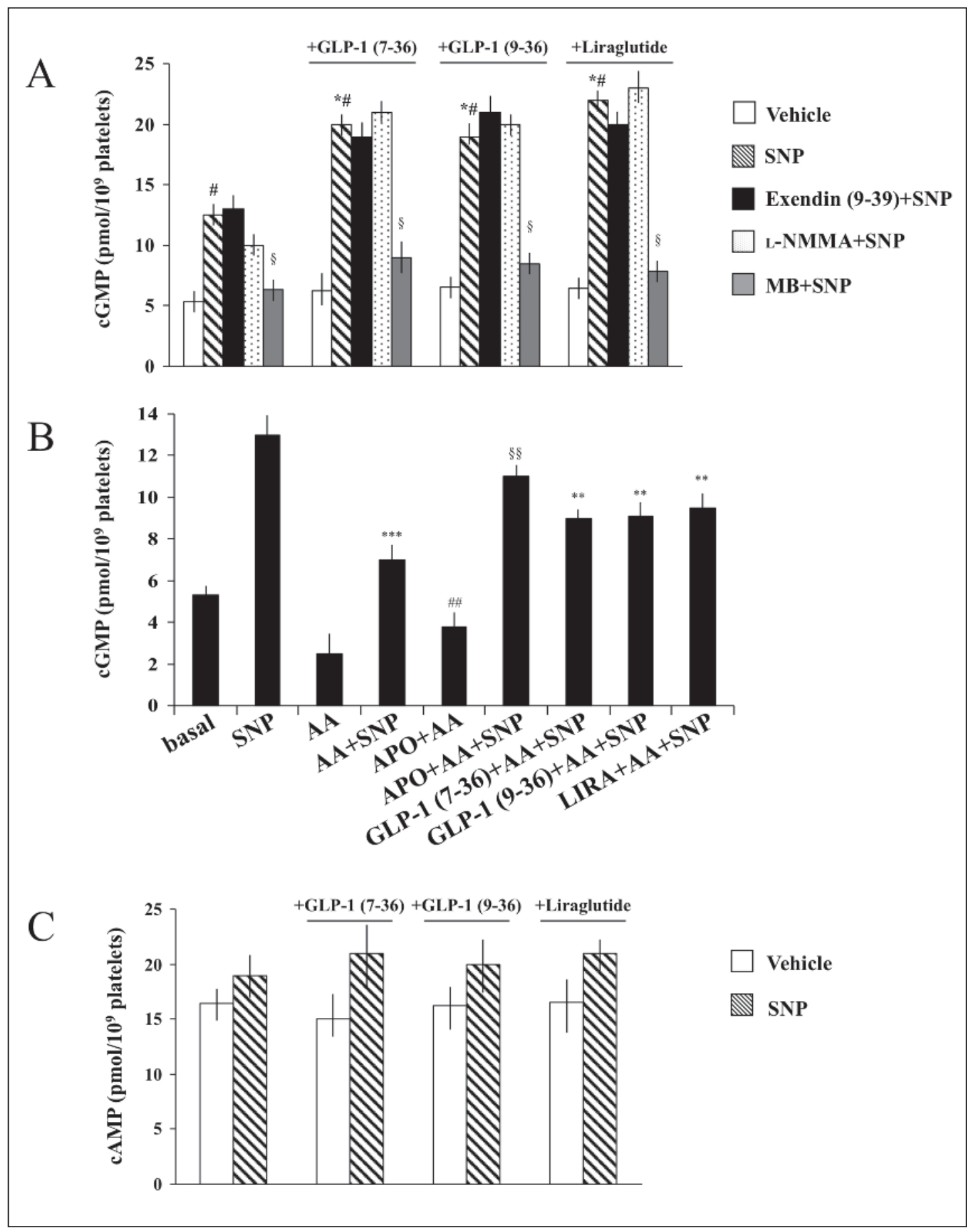

Figure 3: GLP-1 effects on CGMP and CAMP. Platelets were exposed for 15-min to GLP-1(7-36), GLP-1(9-36) and Liraglutide, and their effects were evaluated on the SNP-induced ( $5 \mu \mathrm{mol} / \mathrm{l})$ cGMP levels both in absence and presence of Exendin(9-39) (100 nmol/l), NO-synthase inhibitor L-NMMA (1 mmol/l), sGC inhibitor MB $(50 \mu \mathrm{mol} / \mathrm{l})$ ( $n=12$ per group) $(A)$ or AA $(100 \mu \mathrm{mol} / \mathrm{l})$ and the NADPH-oxidase inhibitor Apocynin $(10 \mu \mathrm{mol} / \mathrm{l})$ $(n=6)(B)$. Platelets were exposed for $15 \mathrm{~min}$ to GLP-1(7-36), GLP-1(9-36) and Liraglutide and their effects were evaluated $(5 \mu \mathrm{mol} / \mathrm{l})$ on cAMP levels both in the absence and presence of SNP $(5 \mu \mathrm{mol} / \mathrm{l})(\mathrm{n}=6)$ (C). ANOVA: $p<0.0001$, Bonferroni's posttest: ${ }^{*} p<0.05$ vs SNP alone, \#p $<0.05$ vs basal, unpaired Student's test: $\S$ $\mathrm{p}<0.01$ vs SNP, ** $\mathrm{p}<0.05$ vs $A A+S N P$, *** $p<0.001$ vs SNP, \#\# $p<0.05$ vs $A A, \S \S p<0.05$ vs $A A+S N P$

\section{GLP-1 influence on cGMP/PKG/VASP pathway activation}

\section{cGMP and CAMP production}

In the presence of IBMX, GLP-1(7-36), GLP-1(9-36) and Liraglutide alone did not significantly modify cGMP $(n=12 ; \mathrm{p}=\mathrm{NS}$ vs basal values for each GLP-1 receptor agonist) ( Figure 3A) or cAMP concentrations ( $n=6$; $p=N S$ vs basal values for each GLP-1 receptor agonist) ( Figure 3C). However, the cGMP synthesis induced by SNP was significantly enhanced by a 15 -min preincubation with GLP-1(7-36), GLP-1(9-36) and Liraglutide, whereas cAMP levels showed a trend to increase in response to SNP+GLP-1 peptides, while not reaching statistical significance $(n=6 ; p=N S$ vs basal values for each GLP-1 receptor agonist with or without SNP).

Furthermore, the effects of GLP-1(7-36), GLP-1(9-36) and Liraglutide on NO-induced cGMP production: i) are not mediated by 


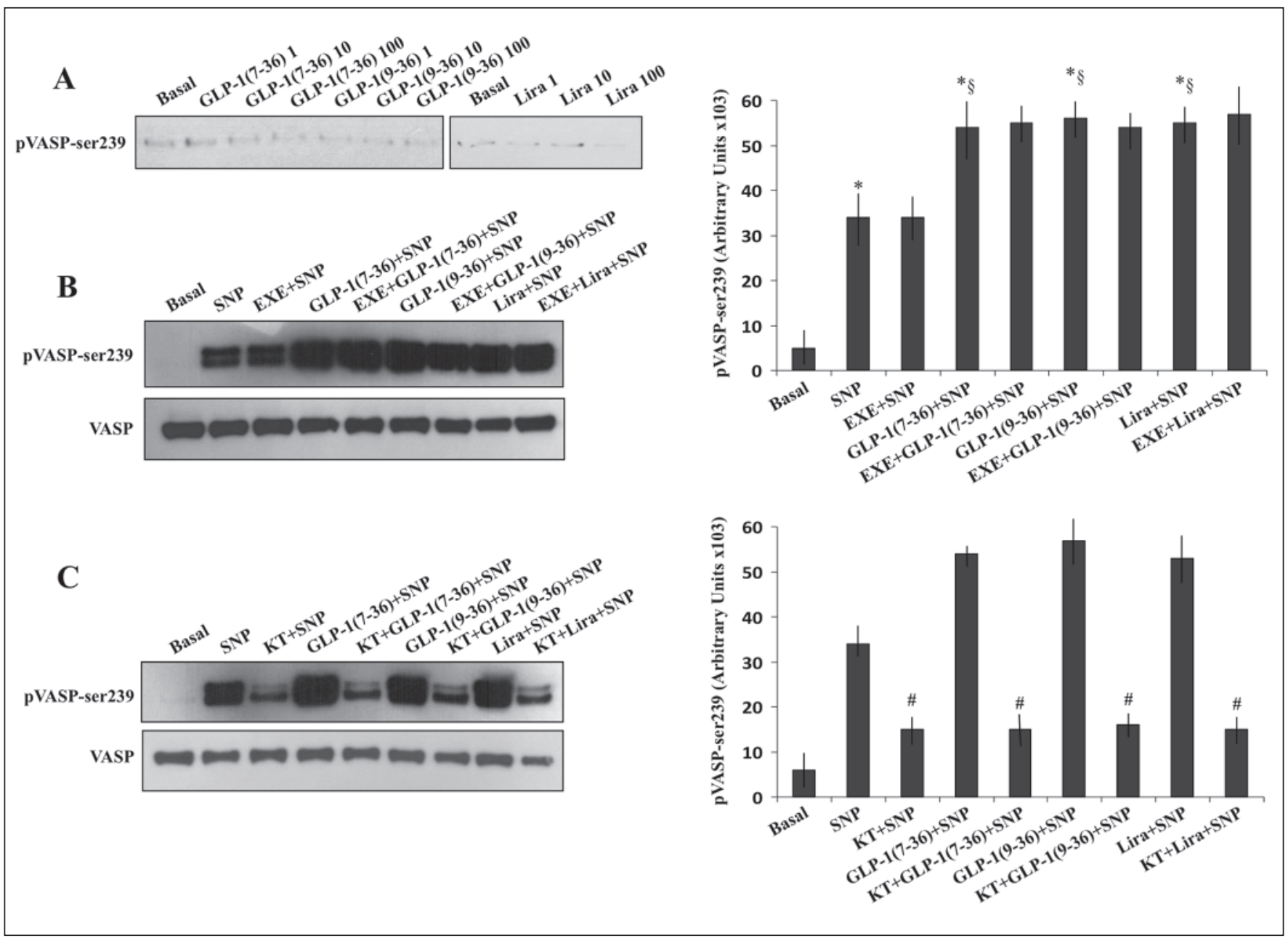

Figure 4: GLP-1 effects on VASP phosphorylation. Dose-dependent effects of a 15-min preincubation with GLP-1(7-36), GLP-1(9-36) and Liraglutide (1-10-100 nmol/l) on VASP phosphorylation at Serine-239 $(A)$ and influence of a 15-min preincubation with GLP-1(7-36), GLP-1(9-36) and Liraglutide $(100 \mathrm{nmol} / \mathrm{l})$ on the SNP-induced VASP phosphorylation at Serine-239 in the absence or in the presence of Exendin(9-39) (100 nmol/l) (B) and the PKG inhibitor KT5823 ( $1 \mu \mathrm{mol} / \mathrm{l})$ (C) ( $n=10$ for all). ANOVA: $p<0.0001$, Bonferroni's post-test: ${ }^{*} p<0.05$ vs basal, $\S p<0.05$ vs SNP alone; Unpaired Student's test: $\# p<0.0001$ vs SNP alone or GLP-1 peptides+SNP.
GLP-1R, since cGMP levels did not differ in the presence of Exen$\operatorname{din}(9-39)(\mathrm{n}=12, \mathrm{p}=\mathrm{NS}$ for each); ii) are not mediated by NOS activity, since cGMP levels were not different in the presence of the NOS inhibitor L-NMMA ( $\mathrm{n}=12, \mathrm{p}=\mathrm{NS}$ for each); iii) are dependent on sGC activity, since the sGC inhibitor MB suppressed cGMP levels induced by SNP alone ( $\mathrm{n}=12$, vs basal values $\mathrm{p}=\mathrm{NS}$ for each) and SNP + GLP-1 peptides ( $\mathrm{n}=12$, vs SNP alone $\mathrm{p}=\mathrm{NS}$ for each).

To clarify whether GLP-1 peptides can influence the NO-induced cGMP production in conditions of augmented oxidative stress, platelets were preincubated with GLP-1 peptides, challenged with the proaggregant agent AA and stimulated by SNP $(\nabla$ Figure 3B). AA decreased the SNP-induced cGMP production $(46 \%)$ and GLP-1 (7-36), GLP-1 (9-36) and Liraglutide produced a partial recovery $(28 \%, 31 \%$ and $35 \%$, respectively). Since AA is known to activate NADPH-oxidase, the most important intracellular source of superoxide anion, we tested the influence of the NADPH-oxidase inhibitor apocynin $(10 \mu \mathrm{mol} / \mathrm{l})$ on the reduction of NO-induced cGMP production exerted by AA. The preincubation with apocynin determined a partial recovery of cGMP levels measured in the presence of AA alone $(50 \%)$ or $\mathrm{AA}+\mathrm{SNP}$ (57\%).

In the absence of IBMX, GLP-1 related peptides alone did not significantly modify cGMP concentrations ( $\mathrm{pmol} / 10^{9}$ platelets) (from $3.5 \pm 0.5$ to $4.2 \pm 0.5$ with GLP-1(7-36), to $3.8 \pm 0.1$ with GLP-1(9-36) and to $4.0 \pm 0.5$ with Liraglutide; $n=6, p=N S$ vs basal values for each). GLP-1 related peptides preserved their ability to increase cGMP levels stimulated by SNP: from $6.3 \pm 0.5$ with SNP $(\mathrm{n}=6$; ANOVA: $\mathrm{p}<0.0001$; Bonferroni's post-hoc test: $\mathrm{p}<0.05$ vs basal values) to $9.2 \pm 0.6$ with GLP-1(7-36)+SNP, to $9.8 \pm 0.4$ with GLP-1(9-36)+SNP and to $9.5 \pm 0.5$ with Liraglutide+SNP ( $\mathrm{n}=6$; ANOVA: $p<0.0001$; Bonferroni's post-hoc test: $p<0.05$ vs SNP alone for each). cAMP levels were not affected by SNP, GLP-1 peptides and SNP+GLP-1 peptides (data not shown). 


\section{VASP phosphorylation at Serine-239}

GLP-1(7-36), GLP-1(9-36) or Liraglutide modify per se the amount of VASP phosphorylated at Serine-239 $(n=10$, vs baseline $\mathrm{p}=\mathrm{NS})$ neither at concentrations lower than $100 \mathrm{nmol} / \mathrm{l}(1-10$ nmol/l) ( Figure 4A) nor at shorter time points (data not shown). In the presence of SNP $(n=10)$ a significant increase of pVASP-ser239 was observed and this was enhanced by GLP-1(7-36), GLP-1(9-36) and Liraglutide ( Figure 4B, C). Quantitatively, after exposure to SNP, GLP-1(7-36), GLP-1(9-36) and Liraglutide further increased the SNP-induced pVASP by $44 \pm$ $5 \%, 51 \pm 7 \%$ and $41 \pm 8 \%$, respectively, without significant differences among GLP-1 peptides.

The preincubation with the PKG inhibitor KT5823 significantly reduced pVASP induced by SNP with GLP-1 peptides, whereas GLP-1(7-36), GLP-1(9-36) and Liraglutide effects did not differ in the presence of Exendin(9-39) (vs SNP+GLP-1 peptides p=NS).

\section{GLP-1 influence on Collagen- and AA-induced phosphorylation of Akt and ERK-1/2}

GLP-1(7-36), GLP-1(9-36) or Liraglutide (n=10) similarly reduced the increased pAkt and pERK-2 amounts induced by collagen ( Figure 5) or AA ( Figure 6). These effects were influenced neither by Exendin(9-39)(vs collagen+GLP-1 or $\mathrm{AA}+\mathrm{GLP}-1$ peptides $\mathrm{p}=\mathrm{NS}$ ) nor by the PKA inhibitor PKI (vs collagen+GLP-1 or AA+GLP-1 peptides $\mathrm{p}=\mathrm{NS}$ ), thus indicating that GLP-1(7-36), GLP-1(9-36) or Liraglutide effects on pAkt and pERK-2 are not mediated by GLP-1R and do not involve PKA activation. Conversely, the effect of each GLP-1-related peptide was reduced after preincubation with KT5823, suggesting the involvement of PKG in the ability of GLP-1 to interfere with the activation of PI3-K/Akt and MAPK/ERK-2 pathways stimulated by collagen or AA.

\section{GLP-1 influence on ROS production}

As shown in $>$ Figure 7, stimulation with $100 \mu \mathrm{mol} / \mathrm{l} \mathrm{AA}$ led to an eight-fold increase in intracellular ROS production versus basal values $(\mathrm{n}=12, \mathrm{p}<0.0001)$. Our results showed that $A A$-induced ROS production: i) involves $\mathrm{PI} 3-\mathrm{K}$, since, in comparison to AA alone, wortmannin and LY294002 reduced ROS generation to a similar extent; ii) is more strictly dependent on the MAPK/ERK pathway because almost completely inhibited by U0126; iii) is at least partly mediated by NADPH-oxidase activity because similarly inhibited by apocynin and DPI ( Figure 7A).

When we investigated the influence of GLP-1 on ROS synthesis, we found a reduction by $32 \pm 4 \%$ with GLP-1(7-36) ( $>$ Figure 7B), $38 \pm 7 \%$ with GLP-1(9-36) ( Figure 7C) and $40 \pm 9 \%$ with Liraglutide ( $>$ Figure 7D) $(\mathrm{n}=12)$ without significant differences among the GLP-1 peptides. Similar results were obtained when we measured DHE oxydation as marker of superoxide production: AA induced an increase on basal values of superoxide concentrations by $6 \pm 1(\mathrm{p}<0.0001, \mathrm{n}=6)$ that was reduced by $33 \pm$ $9 \%$ with GLP-1 $(7-36)(\mathrm{p}<0.001, \mathrm{n}=6)$, by $38 \pm 11 \%$ with GLP-1
(9-36) $(\mathrm{p}<0.005, \mathrm{n}=6)$ and by $40 \pm 13 \%$ with Liraglutide $(\mathrm{p}<0.001$, $\mathrm{n}=6)$.

In order to verify the linkage between the inhibition of PI3-K, ERK-1/2 and NADPH-oxidase and GLP-1 effects on ROS, platelets were pre-treated with the mentioned above inhibitors, incubated with GLP-1(7-36), GLP-1(9-36), or Liraglutide and the ROS generation measured. Data indicate that the ability of GLP-1(7-36), GLP-1(9-36), or Liraglutide to further inhibit ROS production was lost in the presence of every inhibitor, thus suggesting a complex signalling network regulating ROS production and influenced by the GLP-1-related peptide action.

\section{Discussion}

The main findings of this study were that platelet exposure to GLP-1: i) induced an increase of the NO-donor SNP ability to inhibit platelet aggregation, to stimulate cGMP production and to enhance the extent of phosphorylation of VASP-ser239; ii) reduced the activation of PI3-K/Akt and MAPK/ERK-2 pathways probably through a PKG-dependent mechanism; iii) reduced ROS production. These results were similarly obtained in the presence of the native form GLP-1(7-36), the truncated peptide GLP-1(9-36), and the GLP-1 analogue Liraglutide.

This study describes an association between GLP- 1 and platelet function by adding further information on GLP effects on the cardiovascular system.

The main effects of GLP-1 on its target tissues, such as pancreatic islets, are mediated by GLP-1R. Human megakaryocytes express a functional GLP-1R (13) and in our study by western blot and flow cytometry assays we found that GLP-1R is also expressed on platelets; thus, we could hypothesise that the GLP-1R-dependent pathway may be involved in the platelet effects of GLP-1(7-36). However, we observed that when platelets were preincubated with Exendin(9-39), which functions as a specific GLP-1R antagonist, GLP-1 effects were preserved not only for GLP-1(9-36) but also for GLP-1(7-36) and Liraglutide, suggesting for both molecules a GLP-1R independent signalling. Furthermore, in our study the effects of GLP-1 on the investigated aspects of platelet responses were not mediated by cAMP signalling, which is generally involved in the GLP-1R-mediated actions of GLP-1 (22). Our proposed schematic signalling involved in the GLP-1(7-36), GLP-1(9-36) and Liraglutide effects on platelets is summarised in $>$ Figure 8 .

From the biochemical point of view, our original data show that platelet exposure to GLP-1 (7-36), GLP- (9-36) or Liraglutide even though alone does not exert direct effects on NO signalling, it increases the NO-induced activation of the cGMP/PKG/VASP pathway. In particular, we found that each GLP-1-related peptide amplifies to a similar extent the exogenous NO capability to inhibit platelet aggregation induced by different agonists; a possible explanation of this phenomenon could be an augmentation of NO-induced cGMP synthesis and consequent PKG activity, as mirrored by the increased levels of pVASP-ser239. Previous data suggested that GLP-1 can up-regulate endothelial NOS (eNOS) ac- 


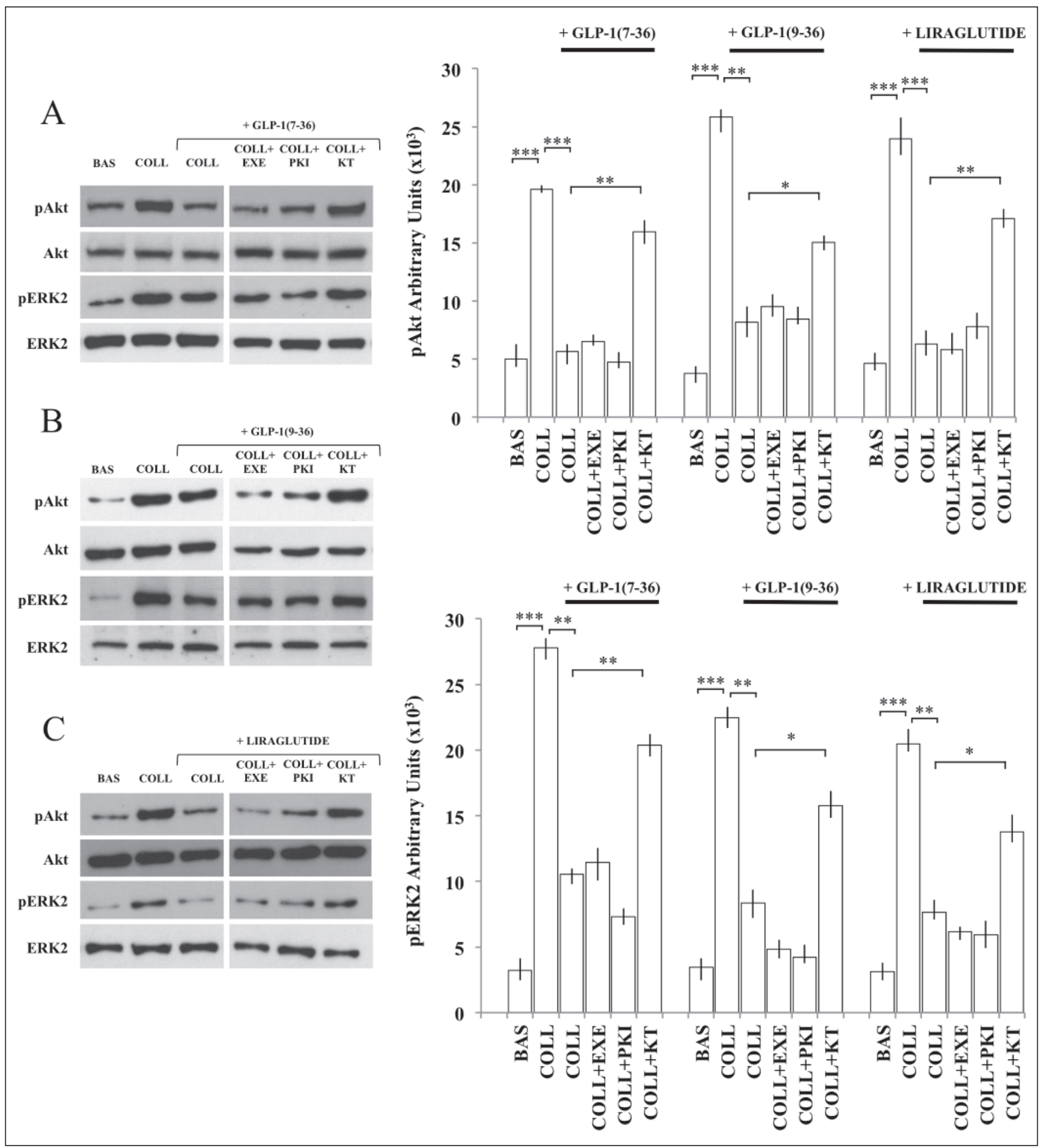

Figure 5: GLP-1 effects on AKT and ERK-2 signalling. Platelets were exposed for 15 min to GLP-1(7-36) (A), GLP-1(9-36) (B) and Liraglutide (C) $(100 \mathrm{nmol} / \mathrm{l})$ and evaluated for Akt and ERK-1/2 phosphorylation after stimulation with collagen in the absence or in the presence of Exendin(9-39) (Exe)
$(100 \mathrm{nmol} / \mathrm{l})$, the PKA inhibitor PKI $(10 \mu \mathrm{mol} / \mathrm{l})$ and the PKG inhibitor KT5823 (KT) $(1 \mu \mathrm{mol} / \mathrm{l})(\mathrm{n}=10 \mathrm{for}$ all). Blots are representative of 10 independent experiments. Unpaired Student's test: ${ }^{*} p<0.05,{ }^{* *} p<0.001,{ }^{* * *} p<0.0001$. 


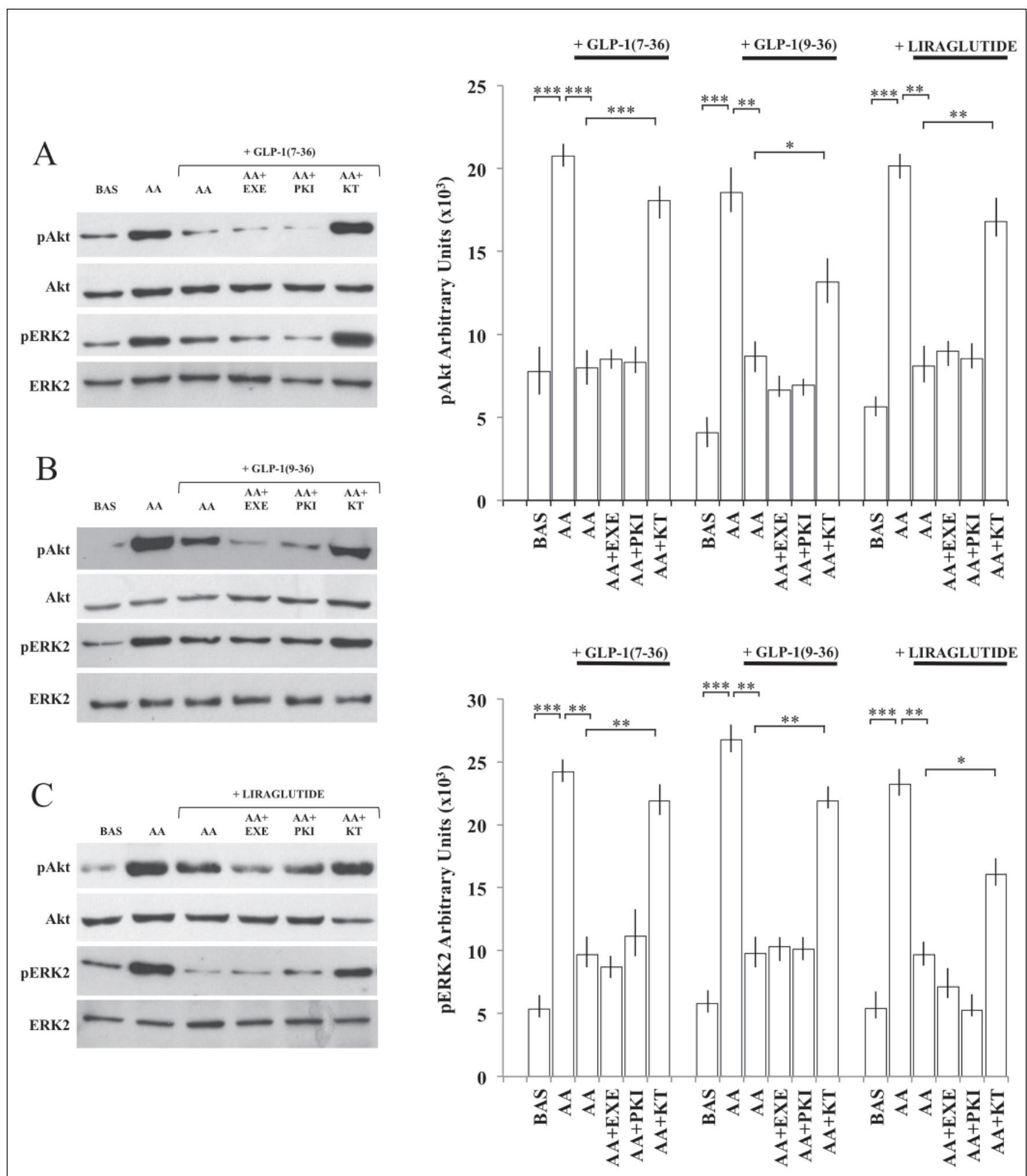

Figure 6: GLP-1 effects on AKT and ERK-2 signalling. Platelets were exposed for 15 min platelet to GLP-1(7-36) (A), GLP-1(9-36) (B) and Liraglutide $(C)$ and evaluated for Akt and ERK-1/2 phosphorylation after stimulation with $A A$ in the absence or in the presence of Exendin(9-39) (Exe) (100 nmol/l), the PKA inhibitor PKI $(10 \mu \mathrm{mol} / \mathrm{l})$ and the PKG inhibitor KT5823 (KT) $(1 \mu \mathrm{mol} / \mathrm{l})(\mathrm{n}=10$ for all). Blots are representative of 10 independent experiments. Unpaired Student's test: ${ }^{*} p<0.01,{ }^{* *} p<0.005,{ }^{* *} p<0.0001$. 
tivity and protein expression through the GLP-1R-dependent and GLP-1(9-36)-related pathways in vascular cells (8); furthermore, the GLP-1-induced NO synthesis and release via eNOS activation can explain the direct vasodilation by GLP-1 (23). Interestingly, Cameron et al. (13) found that the injury-induced thrombus formation in vivo was inhibited by the GLP-1R agonist exenatide although the same authors did not exclude the possibility that these effects could be mediated by endothelium. Surprisingly, they also demonstrated that exenatide - but not GLP-1 (7-36) - exerts antiaggregating effects on platelets without explaining the discrepancy between these peptides which show similar affinity for GLP-1R. In our study, Liraglutide alone did not show any antiaggregating effect to AA and collagen.

In our study, we did not evaluate the direct influence of GLP-1 on NOS activity. Thus, we cannot exclude a GLP-1 effect on NO synthesis. However, GLP-1(7-36), GLP-1(9-36) and Liraglutide per se did not significantly increase platelet cGMP and pVASPser239 levels and their ability to enhance NO action on cGMP/ PKG/VASP pathway was not affected by the NOS inhibitor L-NMMA.

The ability of GLP-1(7-36), GLP-1(9-36) and Liraglutide to increase the antiaggregating effects of $\mathrm{NO}$ was not restricted to stimulation by one agonist, but involved all platelet activators used in our study. This fact implies that the target site of GLP-1 was shared among many signalling pathways, rather than specific for a particular stimulant.

Present data show that GLP-1(7-36), GLP-1(9-36) or Liraglutide significantly decreased the ROS production induced by AA which normally generates a variety of ROS essential for maximal platelet activation (24).

ROS generated by platelet activating agents act as second messengers and might affect platelet function by reacting with NO. Therefore, ROS may partially modulate platelet function by attenuating $\mathrm{NO}$ bioavailability via $\mathrm{ONOO}^{-}$generation (25) thus reducing the activation of the cGMP/PKG/VASP pathway. The finding that in our study GLP-1 attenuates oxidative stress and enhances the activation of cGMP/PKG/VASP by exogenous NO can

\section{What is known about this topic?}

- Subjects affected by type 2 diabetes receiving GLP-1-based therapy show a decreased number of cardiovascular events.

- The GLP-1 analogue exenatide exerts antiaggregating effects.

- Megakariocyte cells express GLP-1 receptor.

\section{What does this paper add?}

- Human platelets express GLP-1 receptor.

- The bioactive native GLP-1(7-36)amide, the GLP-1 analogue Liraglutide but also the dipeptidyl peptidase 4-induced degradation product GLP-1(9-36)amide increase nitric oxide/cGMP/PKG/VASP and decrease PI/3K and MAPK/ERK-2 pathway activation in human platelets.

- GLP-1-related peptides reduce platelet oxidative stress.

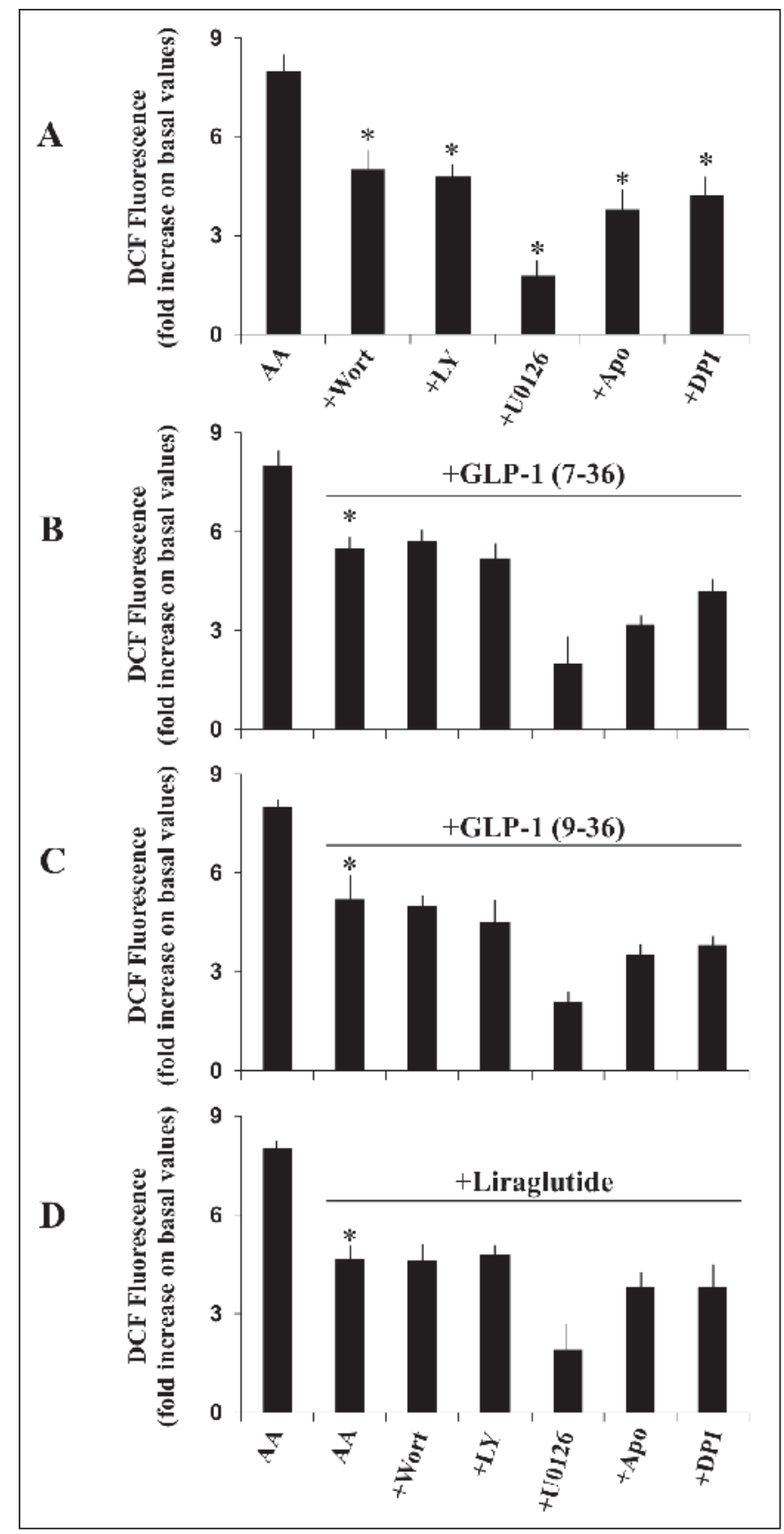

Figure 7: GLP-1 effects on ROS production. ROS measurement (DCF-DA assay) in platelets stimulated by $A A(100 \mu \mathrm{mol} / /)$ in the absence and in the presence of a 20-min preincubation with: PI3-K inhibitors Wortmannin (100 nmol/l)

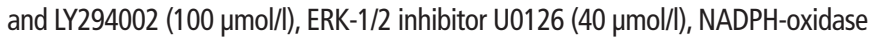
inhibitors apocynin $(10 \mu \mathrm{mol} / \mathrm{l})$ and DPI $(10 \mu \mathrm{mol} / \mathrm{l})(\mathrm{n}=12)(\mathrm{A})$. ANOVA: $p<0.0001$, Bonferroni's post-test: * $p<0.5$ vs AA alone. Effects of a 15 -min preincubation with GLP-1(7-36) (B), GLP-1(9-36) (C) and Liraglutide (100 nmol/I) (D) on platelet ROS production (DCF-DA assay) stimulated by AA $(100 \mu \mathrm{mol} / \mathrm{l})(\mathrm{n}=6$ for all) in the absence and in the presence of a-20 min preincubation with Wortmannin (100

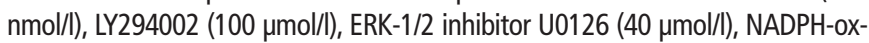
idase inhibitors apocynin $(10 \mu \mathrm{mol} / \mathrm{l})$ and DPI $(10 \mu \mathrm{mol} / \mathrm{l})$. ANOVA: $p<0.0001$, Bonferroni's post-test: ${ }^{*}<<0.5$ vs AA alone; unpaired Student's test: Wortmannin vs Wortmannin+GLP-1-related peptides, LY294002 vs LY294002+GLP-1-related peptides, U0126 vs U0126+GLP-1 related peptides, Apocynin vs Apocynin+GLP-1 related peptides, DPI vs, DPI+GLP-1 related peptides $=$ NS. 


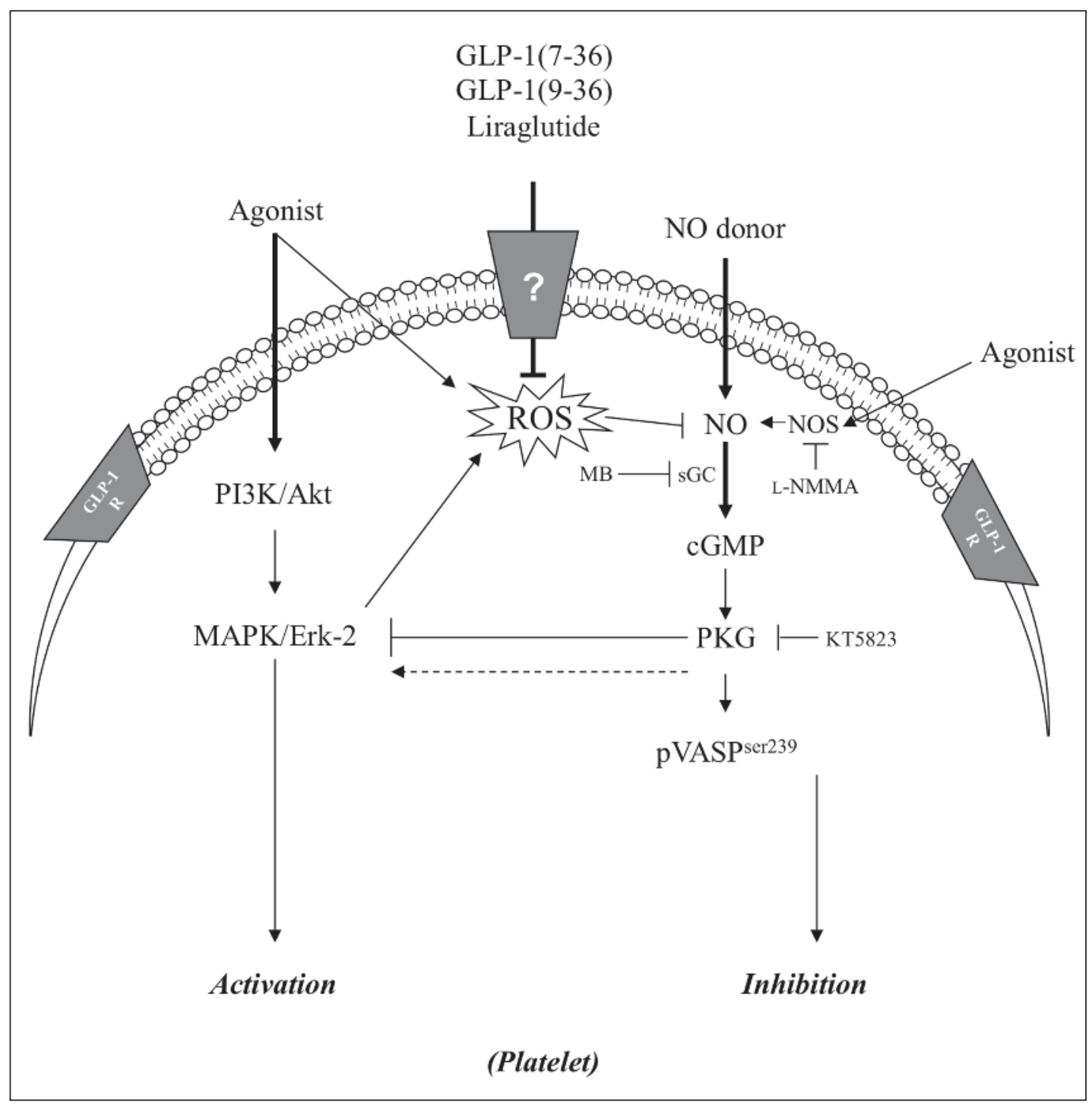

Figure 8: Schematic of proposed signalling involved in GLP-1 effects on platelets. The scheme shows the GLP1-related peptides ability to influence platelet function by improving the NO inhibitory effects and attenuating, via a PKG-mediated mechanism, the activation of PI3-K and MAPK signalling stimulated by agonists. In this scenario, a role can be played by GLP-1 ability to interfere with the stimulant-induced ROS production and all these effects are independent of the known GLP-1 receptor (GLP-1R) which is expressed on platelet surface.

induce to hypothesize that, in the last phenomenon, a role may be played by the increased NO bioavailability due to GLP-1-mediated ROS reduction.

We observed that the AA-induced increase of ROS was reduced by the ERK-1/2 inhibitor U0126, the PI3-K inhibitors wortmannin and LY294002, and the NOX inhibitors apocynin and DPI thus demonstrating a complex signalling networks, regulating ROS production. Noteworthy, ROS synthesis was almost completely blocked by the ERK-1/2 inhibitor U0126, to indicate that the MAPK/ERK- $1 / 2$ pathway is one of the major players in intracellular production of ROS by AA. Nevertheless, when using the mentioned above specific inhibitors in the presence of GLP-1, their inhibitory effects on ROS production were not enhanced, thus suggesting a putative role for MAPK/ERK, and an involvement of the PI3-K and NADPH-oxidase pathways on the capability of GLP-1 related peptides to reduce ROS production.

When we investigated the influence of GLP-1 on the agonist-induced PI3-K/Akt and MAPK/ERK-1/2 signalling activation, we found that GLP-1(7-36), GLP-1(9-36) and Liraglutide reduced to a similar extent both Akt and ERK-2 activation stimulated by AA or collagen.

Altogether, MAPK/ERK-1/2 and PI3-K/Akt regulate platelet functions $(26,27)$ even if the role of MAPK/ERK-1/2 activation pathway on platelet aggregation is controversial (28). Our findings also confirmed the evidence of a crosstalk between PI3-K/Akt and ERK-2 activated by collagen and AA since the PI3-K inhibitor wortmannin abolished not only Akt but also ERK-2 phosphorylation (data not shown).

We found that the effects of GLP-1(7-36), GLP-1(9-36) and Liraglutide on the agonist-induced pAkt and pERK-2 levels were significantly reduced by the PKG inhibitor KT5823 whereas they were not affected by the PKA inhibitor PKI. These data could suggest that GLP-1 effects are mediated by PKG whereas the adenylate cyclase-cAMP-PKA pathway is not involved.

To explain the relationship between the PI3-K and MAPK pathways and $\mathrm{PKG}$, it is important to underline that an activation 


\section{Abbreviations}

AA, Arachidonic acid; APC, Allophycocyanin; CEPI, Collagen/epinephrine cartridge; cGMP, Guanosine-3',5'-cyclic monophosphate; cAMP, Adenosine-3', 5'-cyclic monophosphate; DCF-DA, 2'-7'-dihydrodichlorofluoresceine diacetate; DHE, Dihydroethidium; DPI, Diphenyleneiodonium; DPP-4, Dipeptidyl peptidase-4; GC, Guanylate cyclase; GLP-1, Glucagon-like peptide 1; GLP-1R, Glucagon-like peptide 1 receptor; IBMX, 3-isobutyl-1-methylxanthine; L-NMMA, NG-monomethyl-L-arginine; MA, Maximal aggregation; MAPK, Mitogen activated protein kinase; MB, Methylene blue; NO, Nitric oxide; NOS, NO synthase; NOX, NADPH-oxidase; PE, Phycoerythrin; PI3-K, Phosphatidylinositol-3-kinase; PKA, Protein kinase A; PKG, cGMP-dependent protein kinase; PRP, Platelet-rich plasma; ROS, Reactive oxygen species; SNP, Sodium nitroprusside; TXA 2 , Thromboxane $A_{2}$; VASP, Vasodilator-stimulated phosphoprotein; WB, Whole blood; WP, Washed platelets.

of sGC may also be directed by agonists (29). Actually, it has been described that NO may play a biphasic role in platelet reactivity: at low concentrations, such as those induced by platelet eNOS stimulated by agonists, NO promotes platelet activating signalling, whereas at high concentrations, such as those released by the NO donor SNP at micromolar levels, it induces a second phase of cGMP signalling which results in platelet inhibition (30). Thus, we can hypothesise that, when platelets are stimulated by physiological agonists in conditions of reduced NO breakdown, like in the presence of GLP-1, endogenous NO levels and PKG activity increase, thus promoting PKG inhibitory effects. However, this hypothesis needs to be confirmed by more extensive experiments.

In our study, the effects of GLP-1(7-36) and GLP-1(9-36) were very similar to those observed with Liraglutide, and also in the last case the pathway involved could be GLP-1(9-36)-related. Actually, data from Literature suggest that GLP-1(9-36) is responsible for some anti-atherogenic effects of Liraglutide rather than the intact Liraglutide (30). It is likely that the beneficial effects of Liraglutide might be mediated through a distinct receptor for GLP-1(9-36) also in our study. Interestingly, recent data from the LEADER trial have demonstrated that patients with type 2 diabetes taking Liraglutide and at high risk for cardiovascular events had lower rates of cardiovascular events and death from any cause than did those in the placebo group (31). We can speculate that the beneficial effects of Liraglutide on platelets could contribute to these results.

In the present study, we investigated platelets from normal subjects and consequently we did not give information of the situation in diabetes mellitus. Further study carried out in high glucose condition as well as with platelets from subjects with diabetes are needed to explore the relationship between GLP-1 stimulated platelets and vessel abnormalities in diabetes.

At the moment, we can only suppose that a reduced production or synthesis of GLP-1 may contribute to reduce platelet sensitivity to powerful antiaggregating agents such as NO.
In conclusion, collectively, this study reports that the GLP-1 directly influences platelet function and suggests a potential protective role on the cardiovascular system.

\section{Conflicts of interest}

None declared.

\section{References}

1. Ussher JR, Drucker DJ. Cardiovascular biology of the incretin system. Endocr Rev 2012; 33: 187-215.

2. Toft-Nielsen MB, Damholt MB, Madsbad S, et al. Determinants of the impaired secretion of glucagon-like peptide-1 in type 2 diabetic patients. J Clin Endocrinol Metab 2001; 86: 3717-3723.

3. Abu-Hamdah R, Rabiee A, Meneilly GS, et al. The extrapancreatic effects of glucagon-like peptide-1 and related peptides. J Clin Endocrinol Metab 2009; 94: $1843-1852$.

4. Nikolaidis LA, Elahi D, Shen YT, et al. Active metabolite of GLP-1 mediates myocardial glucose uptake and improves left ventricular performance in conscious dogs with dilated cardiomyopathy. Am J Physiol Heart Circ Physiol 2005; 289: H2401-H2408.

5. Nathanson D, Erdogdu O, Pernow J, et al. Endothelial dysfunction induced by triglycerides is not restored by exenatide in rat conduit arteries ex vivo. Regul Pept 2009; 157: 8-13.

6. Ban K, Noyan-Ashraf MH, Hoefer J, et al. Cardioprotective and vasodilatory actions of glucagon-like peptide 1 receptor are mediated through both glucagonlike peptide 1 receptor-dependent and -independent pathways. Circulation 2008; 117: 2340-2350.

7. Ban K, Kim KH, Cho CK, et al. Glucagon-like peptide (GLP)-1(9-36)amidemediated cytoprotection is blocked by exendin(9-39) yet does not require the known GLP-1 receptor. Endocrinology 2010; 151: 1520-1531.

8. Ding L, Zhang J. Glucagon-like peptide-1 activates endothelial nitric oxide synthase in human umbilical vein endothelial cells. Acta Pharmacol Sin 2012; 33: $75-81$.

9. Batchuluun B, Inoguchi T, Sonoda N, et al. Metformin and liraglutide ameliorate high glucose-induced oxidative stress via inhibition of PKC-NAD $(\mathrm{P}) \mathrm{H}$ oxidase pathway in human aortic endothelial cells. Atherosclerosis 2014; 232 : 156-164.

10. Davi G, Patrono C. Mechanisms of disease: platelet activation and atherothrombosis. N Engl J Med 2007; 357: 2482-2494.

11. Vinik AI, Erbas T, Park TS, et al. Platelet dysfunction in type 2 diabetes. Diabetes Care 2001; 24: 1476-1485.

12. Gkaliagkousi E, Ritter J, Ferro A. Platelet-derived nitric oxide signaling and regulation. Circ Res 2007; 101: 654-662.

13. Cameron-Vendrig A, Reheman A, Siraj MA, et al. Glucagon-Like Peptide 1 Receptor Activation Attenuates Platelet Aggregation and Thrombosis. Diabetes 2016; 65: 1714-1723.

14. Scirica BM, Bhatt DL, Braunwald E, et al. Saxagliptin and cardiovascular outcomes in patients with type 2 diabetes mellitus. N Engl J Med 2013; 369: $1317-1326$.

15. White WB, Cannon CP, Heller SR, et al. Alogliptin after acute coronary syndrome in patients with type 2 diabetes. N Engl J Med 2013; 369: 1327-1335.

16. Jacobsen LV, Hindsberger C, Robson, et al. Effect of renal impairment on the pharmacokinetics of the GLP-1 analogue liraglutide. Br J Clin Pharmacol 2009; 68: 898-905.

17. Russo I, Del Mese P, Doronzo G, et al. Platelet resistance to the antiaggregatory cyclic nucleotides in central obesity involves reduced phosphorylation of vasodilator-stimulated phosphoprotein. Clin Chem 2007; 53: 1053-1060.

18. Born GVR. Aggregation of blood platelets by adenosine diphosphate and its reversal. Nature 1962; 194: 927-929.

19. Favaloro EJ. Clinical utility of the PFA-100. Semin Thromb Hemost 2008; 34: 709-733.

20. Russo I, Viretto M, Barale C, et al. High glucose inhibits the aspirin-induced activation of the nitric oxide/cGMP/cGMP-dependent protein kinase pathway and does not affect the aspirin-induced inhibition of thromboxane synthesis in human platelets. Diabetes 2012; 61: 2913-2921. 
21. Eruslanov E, Kusmartsev S. Identification of ROS using oxidized DCFDA and flow-cytometry. Methods Mol Biol 2010; 94: 57-72.

22. Doyle ME, Egan JM. Mechanisms of action of glucagon-like peptide 1 in the pancreas. Pharmacol Ther 2007; 113: 546-593.

23. Ban K, Noyan-Ashraf MH, Hoefer J, et al. Cardioprotective and vasodilatory actions of glucagon-like peptide 1 receptor are mediated through both glucagonlike peptide 1 receptor-dependent and -independent pathways. Circulation 2008; 117: 2340-2350.

24. Krötz F, Sohn HY, Gloe T, et al. NAD(P)H oxidase-dependent platelet superoxide anion release increases platelet recruitment. Blood 2002; 100: 917-924.

25. Clutton P, Miermont A, Freedman JE. Regulation of endogenous reactive oxygen species in platelets can reverse aggregation. Arterioscler Thromb Vasc Biol 2004; 24: 187-192.

26. Aharonovitz O, Granot Y. Stimulation of mitogen-activated protein kinase and $\mathrm{Na}+\mathrm{H}+$ exchanger in human platelets. J Biol Chem 1996; 271: 16494-16499.
27. Kovacsovics TJ, Bachelot C, Toker A, et al. Phosphoinositide 3-kinase inhibition spares actin assembly in activating platelets but reverses platelet aggregation. J Biol Chem 1995; 270: 11358-11366.

28. Borsch-Haubold AG, Kramer RM, Watson SP. Inhibition of mitogen-activated protein kinase does not impair primary activation of human platelets. Biochem J 1996; 318: 207-212.

29. Zhang G, Xiang B, Dong A, et al. Biphasic roles for soluble guanylyl cyclase (sGC) in platelet activation. Blood 2011; 118: 3670-3679.

30. Hou J, Manaenko A, Hakon J. Liraglutide, a long-acting GLP-1 mimetic, and its metabolite attenuate inflammation after intracerebral hemorrhage. J Cereb Blood Flow Metab 2012; 32: 2201-2210.

31. Marso SP, Daniels GH, Brown-Frandsen K, et al. Liraglutide and Cardiovascular Outcomes in Type 2 Diabetes. N Engl J Med 2016; 375: 311-322.

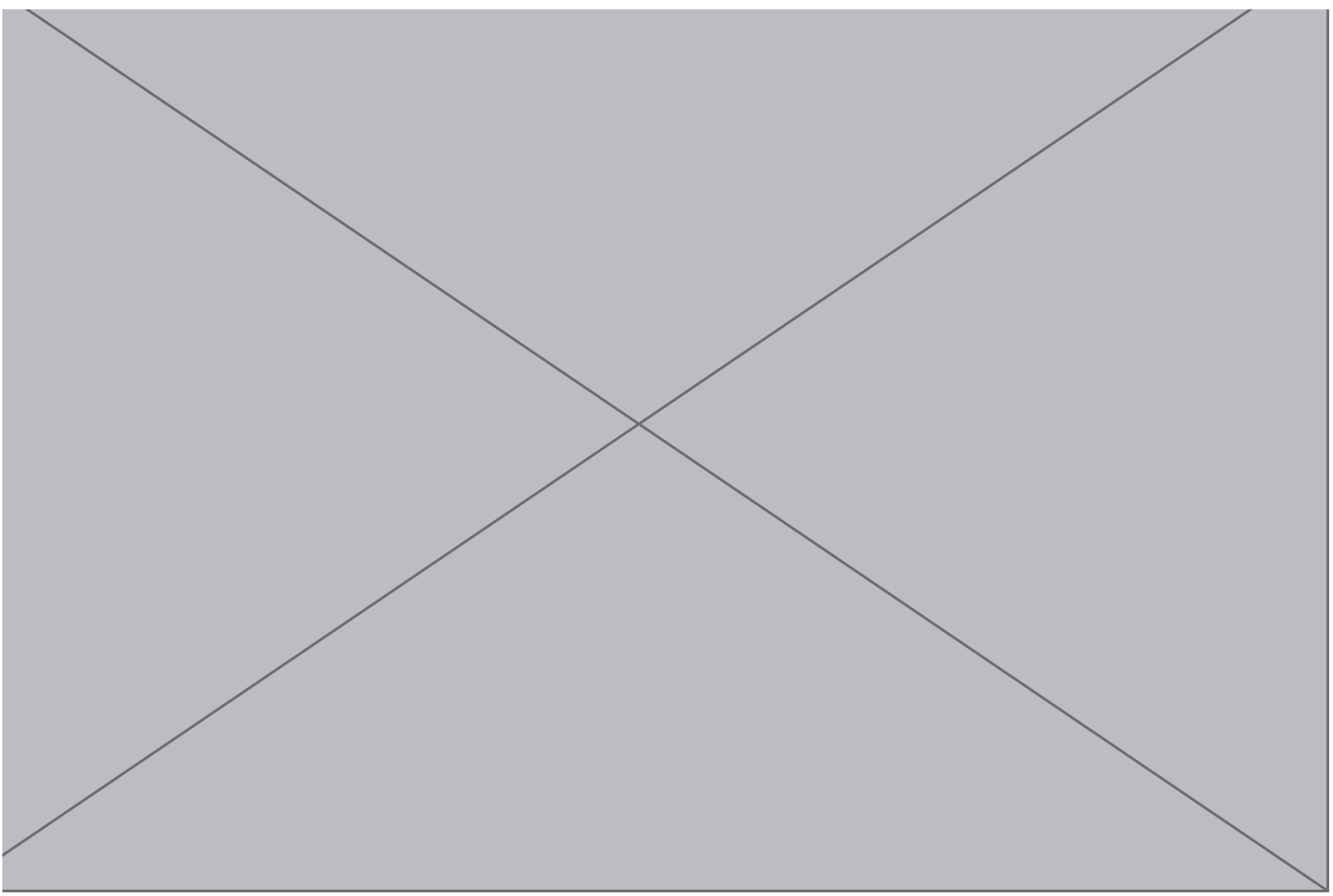

